\title{
REPRESENTATIONS OF INFINITE SOLUBLE GROUPS
}

\author{
by IAN M. MUSSON
}

(Received 16 June, 1981)

1. Introduction. The purpose of this paper is to study the following two questions.

(1) When does the group algebra of a soluble group have infinite dimensional irreducible modules?

(2) When is the group algebra of a torsion free soluble group primitive?

In relation to the first question, Roseblade [13] has proved that if $G$ is a polycyclic group and $k$ an absolute field then all irreducible $k G$-modules are finite dimensional. Here we prove a converse.

THEOREM A. Let $G$ be a finitely generated hyperabelian group which is not polycyclicby-finite. If $k$ is any field, then $k G$ has an infinite dimensional irreducible module.

We remark that B. A. F. Wehrfritz has recently and independently proved a result similar to Theorem A [17].

Recently some progress has been made on the second question. For instance, Roseblade has solved the problem for polycyclic groups in [14], while in [2] Brown has treated the case of torsion free metanilpotent groups whose Fitting subgroup has infinite rank.

Here we study the finite rank case. In many situations a reduction to the case where the group $G$ has an abelian normal subgroup $A$ with $G / A$ infinite cyclic, seems possible, for example [4, Theorem 4.6]. By a result of Passman [11, 9.3.2] the group algebra $k G$ will always be primitive if $\Delta(G)=1$, and the field $k$ is large enough. Thus we are concerned to find techniques which will work over any field.

We can adapt the definition of a plinth given on [11, p. 547] to this situation. If $A$ is a torsion free abelian subgroup of a group $G$, and $A$ has finite rank, we say $A$ is a plinth in $G$ if there is a subgroup $G_{0}$ containing $A$ such that

(i) $A \leqslant G_{0}$

(ii) $\left|G: G_{0}\right|<\infty$ and $G_{0} / C_{G_{0}}(A)$ is abelian, and

(iii) $G_{0}$ and all its subgroups of finite index act rationally irreducibly on $A$.

If $\Gamma$ is a group of automorphisms of the abelian group $A$, we say $A$ is a $\Gamma$-plinth if $A$ is a plinth in the split extension $G=A \sqsupset \Gamma$.

Suppose that $A$ is an $\langle x\rangle$-plinth and $G=\langle A, x\rangle$. The problem of deciding when $k G$ is primitive here splits into two cases. Suppose that $A_{0}$ is a finitely generated subgroup of $A$ of maximum rank. If $A_{0}^{\langle x\rangle} / A_{0}$ is finite then we may assume $A_{0}=A_{0}^{\langle x\rangle}$. In this case $G$ is locally polycyclic and the methods of [14] can be applied, at least if $k$ is non-absolute (see Corollary 4.6). We study the contrary case where $A_{0}^{\langle x\rangle} / A_{0}$ is infinite.

THEOREM B. Suppose that $A$ is an $\langle x\rangle$-plinth such that $G=\langle A, x\rangle$ is not locally polycyclic. If $k$ is any field, then $k G$ is primitive.

To prove Theorem B, we need a variant of Bergman's Theorem [1], [11, Chapter 9],

Glasgow Math. J. 24 (1983) 43-52. 
for these groups. We prove that if $A$ is an $\langle x\rangle$-plinth and $I$ is a non-zero ideal of $k A$ such that $I^{x}=I$, then $k A / I$ is algebraic (Corollary 4.3). We next look for maximal ideals $M$ of $k A$ such that

$$
\bigcap_{n \in \mathbb{Z}} M^{x^{n}}=0 .
$$

This is closely related to the Ergodic Conjecture of Farkas and Passman [4] and is known to imply primitivity.

We set $M \dagger=A \cap(1+M)$.

Theorem C. Suppose that $A$ is an $\langle x\rangle$-plinth such that $A=A_{0}^{\langle x\rangle}$ for a finitely generated subgroup $A_{0}$ of $A$ and suppose $G=\langle A, x\rangle$ is not polycyclic-by-finite. If $M$ is a maximal ideal of $k A$, then $\bigcap_{n \in \mathbb{Z}} M^{x^{n}}=0$ if and only if $A / M \dagger$ is infinite.

If $A / A_{0}$ is a $p$-group and $k$ is an absolute field of characteristic $p$, then $k A$ has no maximal ideal $M$ with $A / M \dagger$ infinite. However, in this case we deduce Theorem $\mathrm{B}$ from a result of Irving, [8, Theorem 4.2].

Examples of groups satisfying the hypotheses of Theorems $B$ and $C$, may be constructed as follows. Let $x$ be an algebraic number and $A_{0}$ a $\mathbb{Z}$-lattice in $\mathbb{Q}(x), A$ the $\mathbb{Z}\langle x\rangle$-module generated by $A_{0}$, and $G$ the split extension of $A$ by $x$. If we assume that $x^{n}$ does not lie in any of the proper subfields of $\mathbb{Q}(x)$ for $n \geq 1$, then $A$ will be an $\langle x\rangle$-plinth. If in addition we ensure that $x$ is not a unit in the ring of algebraic integers of $\mathbb{Q}(x)$ then $G=\langle A, x\rangle$ is not locally polycyclic.

We remark that Irving has shown that for any integer $r>1$ the group algebra of the group presented by $\left\langle a, x \mid a^{x}=a^{r}\right\rangle$ is primitive over any field, [8, Theorem 5.2].

As it turns out, Theorem $C$ provides more information about $x$-invariant ideals of $k A$. Given any non-zero $x$-invariant ideal $I$ of $k A$, it is easily seen that $\sqrt{I}$ is a semiprime $x$-invariant ideal of $k A$, where $\sqrt{I} / I$ is the nil-radical of $k A / I$. Thus we may assume that $I$ is semiprime. In general, a semiprime ideal of $k A$ is an infinite intersection of prime ideals. However, Theorem $C$ restricts the kinds of maximal ideals $M$ which can contain $I$ to those with $A / M \dagger$ finite.

We prove the following result, which is a sharper version of Corollary 4.3.

TheOREM D. Let $A$ be an $\langle x\rangle$-plinth and I a non-zero $x$-invariant semi-prime ideal of $k A$. Suppose that $A=A_{0}^{(x)}$ where $A_{0}$ is finitely generated, and $A$ is not finitely generated. Then $|A: I \dagger|<\infty$, and $I$ is a finite intersection of maximal ideals.

Theorem $A$ is proved in $\S 3$ of this paper; Theorems $B-D$ in $\S 4$. In $\S 2$, we prove some preliminary results about abelian groups which are needed later.

I am very grateful for the hospitality of the University of Alberta, where this work was carried out.

2. Abelian Groups of Finite Rank. Throughout this section $A$ will denote a torsion free abelian group of finite rank and $A_{0}$ a finitely generated subgroup of maximum rank.

Lemma 2.1. If $M$ is a maximal ideal of $k A$ then $k A / M$ is algebraic over $k$. 
Proof. Since $k A$ is generated by elements which are integral over $k A_{0}, k A$ is integral over $k A_{0}$. Hence by [10, Theorems 44 and 47], $M_{0}=M \cap k A_{0}$ is a maximal ideal of $k A_{0}$. By the Nullstellensatz $k A_{0} / M_{0}$ is finite dimensional and the result follows since $k A / M$ is algebraic over $k A_{0} / M_{0}$.

Lemma 2.2. Every maximal ideal $M$ of $k A$ satisfies $|A: M+|<\infty$ if and only if $k$ is an absolute field of characteristic $p$, and $A / A_{0}$ is a finite extension of a p-group.

Proof. Suppose the stated conditions hold, and that $M$ is a maximal ideal of $k A$. By Lemma $2.1 \mathrm{kA} / \mathrm{M}$ is an absolute field and $A / M^{\dagger}$ is periodic, and so is a finite extension of . a $p$-group. As $k$ has characteristic $p,|A: M \dagger|<\infty$.

To see the necessity of the conditions, let $\tilde{k}$ be the algebraic closure of $k$ and $\phi: A \rightarrow(\tilde{k})^{*}$ a homomorphism from $A$ to the multiplicative group $(\tilde{k})^{*}$ of $\tilde{k}$, and let $F$ be the subfield of $\tilde{k}$ generated by $k$ and $\phi(A)$. Then any element of $F$ may be written as a finite sum $\sum_{x \in A} \lambda_{x} \phi(x)$ where the coefficients $\lambda_{x}$ belong to $k$. We may regard $F$ as a $k A$-module. Suppose that $V$ is a non-zero submodule of $F$ and $\alpha \in V, \alpha \neq 0$. Then $\alpha^{-1}=\sum_{x \in A} \lambda_{x} \phi(x) \in F$. For all $x$ in this finite sum $\alpha \phi(x) \in V$. Therefore $1=\alpha \alpha^{-1}=$ $\sum \lambda_{x} \alpha \phi(x) \in V$. Hence $F$ is an irreducible $k A$-module and $F \cong k A / M$ for some maximal ideal $M$ of $k A$. Clearly $M \dagger=\operatorname{Ker} \phi$.

Now suppose $\zeta$ is an element of $k$ which is not a root of unity and let $a$ be a non-trivial element of $A$. The map $\langle a\rangle \rightarrow k^{*}$ given by $a \rightarrow \zeta$ extends to a homomorphism $\phi: A \rightarrow(k)^{*}$, since $(k)^{*}$ is divisible. As $\operatorname{Ker} \phi \cap\langle a\rangle=1, A / \operatorname{Ker} \phi$ is infinite.

Finally suppose that $A / A_{0} \cong P \oplus Q$ where $P$ is a $p$-group and $Q$ is an infinite $p^{\prime}$-group. Since $Q$ has finite rank, we see that either $Q$ involves some $C_{q^{\infty}}, q \neq p$ or $Q$ involves a direct sum of cyclic groups of prime orders $p_{i}$ for infinitely many primes $p_{i}$. Since $(\tilde{k})^{*}$ contains a copy of each of these groups this yields a homomorphism $\phi: A \rightarrow \tilde{k}^{*}$ such that $A / \operatorname{Ker} \phi$ is infinite.

LEMMA 2.3. Let $x$ be an automorphism of $A$ such that $A=A_{0}^{\langle x\rangle}$. If $C$ is a subgroup of $A$ with $A / C \cong C_{p^{\infty}}$, then there is an element a of $A_{0}$ whose conjugates $\left\{a^{x^{r}} \mid r \in \mathbb{Z}\right\}$ lie in infinitely many distinct cosets of $C$ in $A$.

Proof. Let $A_{0}=\left\langle a_{1}, a_{2}, \ldots, a_{n}\right\rangle$. There is no integer $s$ such that $\left(a_{i}^{x}\right)^{p^{*}} \in C$ for all $i=1, \ldots, n$ and $r \in \mathbb{Z}$, since $A / C$ has unbounded exponent, and $A=A_{0}^{(x)}$ is generated by the conjugates $a_{i}^{x^{\prime}}$. Since there are only finitely many $a_{i}$, there is an element $a=a_{i}$ whose conjugates have unbounded order modulo $C$.

We introduce some notation. Let $B$ be a subgroup of the abelian group $A$ and $p$ a prime. Let $\sqrt{B}$ be the isolator of $B$ in $A$, that is $\sqrt{B} / B$ is the torsion subgroup of $A / B$. Similary we let $\sqrt[p]{B} / B$ and $\sqrt[p^{\prime}]{B} / B$ denote the $p$-torsion and $p^{\prime}$-torsion subgroups of $A / B$ respectively. We say $B$ is isolated, $p$-isolated or $p^{\prime}$-isolated according as $B=\sqrt{B}, B=\sqrt[p]{B}$ or $B=\sqrt[p]{B}$.

LEMMA 2.4. Let $x$ be an automorphism of $A$ such that $A=A_{0}^{\langle x\rangle}$. Suppose $\sqrt[n]{A_{0}} / A_{0}$ is 
infinite. Then $A$ has a subgroup $B$ containing $A_{0}$ such that either

(1) $B^{x} \subsetneq B$ and $B / B^{x}$ is a finite p-group, or

(2) $B \subsetneq B^{x}$ and $B^{x} / B$ is a finite $p$-group.

If in addition $A \otimes_{\mathbb{Z}} \mathbb{Q}$ is irreducible as a $\mathbb{Q}\langle x\rangle$-module, then $\bigcap_{n \in \mathbb{Z}} B^{x^{n}}=1$.

Proof. Let $A_{1}=\sqrt[p]{A_{0}}$ and $A_{2}=\bigcup_{n \geqq 0} A_{0} A_{0}^{x} A_{0}^{x^{2}} \ldots A_{0}^{x^{n}}$. Then $A_{2}^{x} \subseteq A_{2}$. If $a \in A_{1}$, then $a^{m} \in A_{0}$ for some integer $m$ prime to $p$, so $\left(a^{x}\right)^{m} \in A_{0}^{x} \subseteq A_{2} \subseteq A_{1} A_{2}$. Since $A_{1} A_{2}$ is $p^{\prime}$-isolated we have $a^{x} \in A_{1} A_{2}$. Therefore $\left(A_{1} A_{2}\right)^{x} \subseteq A_{1} A_{2}$. If this inclusion is strict, then 1) holds with $B=A_{1} A_{2}$. that

If $\left(A_{1} A_{2}\right)^{x}=A_{1} A_{2}$, then since $A_{0}^{x^{-1}}$ is finitely generated, there is an integer $r$ such

$$
A_{0}^{x^{-1}} \subset A_{1} A_{0} A_{0}^{x} \ldots A_{0}^{x^{r}}=B .
$$

Since $B$ is $p^{\prime}$-isolated this gives $B^{x^{-1}} \subset B$ as before. The inclusion is strict since $\sqrt[p]{A_{0}} / A_{0}$ is infinite, and $B / B^{x^{-1}}$ is a finite $p$-group, so 2 ) holds.

Finally, suppose that $A \otimes_{\mathbb{Z}} \mathbb{Q}$ is irreducible as a $\mathbb{Q}\langle x\rangle$-module and let $C=\bigcap_{n \in \mathbb{Z}} B^{x^{n}}$. Since $C^{x}=C$, either $C=1$ or $C$ has rank $n$.

If $C$ has rank $n$ then $C_{0}=C \cap A_{0}$ has finite index in $A_{0}$. But then $A_{0}^{\langle x\rangle} / C_{0}^{\langle x\rangle}$ has finite exponent and is finite. This implies $A / C$ is finite and so $A / B$ is finite. This is inconsistent with either (1) or (2).

We remark that if $A \otimes_{\mathbb{Z}} \mathbb{Q}$ is irreducible as a $\mathbb{Q}\langle x\rangle$-module, the conclusion of Lemma 2.4 is precisely what is required to apply Theorem 4.2 of Irving's paper [8]. This shows that $k G$ is primitive, where $G=\langle A, x\rangle$ and $k$ is any field of characteristic $p$.

\section{Infinite Dimensional Irreducible Modules}

Lemma 3.1. Let $G$ be a polycyclic-by-finite group, $k$ an absolute field and $M$ a finitely generated $k G$-module of infinite dimension over $k$. Then there are elements $m \in M$ and $x \in G$ such that $m k X$ is infinite dimensional where $X=\langle x\rangle$.

Proof. Without loss of generality $M$ is faithful for $G$ and cyclic, and $M \cong k G / I$ where $I$ is a right ideal of $k G$. Since $G$ must be infinite, it has a torsion free abelian normal subgroup $A=\left\langle a_{1}, \ldots, a_{n}\right\rangle$. If $I \cap k\left\langle a_{j}\right\rangle=0$ for some $j$ then we can take $x=a_{j}$ and $m=1+I \in M$. Otherwise for all $j, k\left\langle a_{j}\right\rangle /\left(I \cap k\left\langle a_{j}\right\rangle\right)$ has finite dimension, and so $k A /(I \cap$ $k A)$ is finite dimensional. Hence, $T=\{a \in A \mid a-1 \in I \cap k A\}$ satisfies $|A: T|<\infty$ since $k$ is absolute (see $[\mathbf{1 1}, 12.3 .8]$ ). Therefore, $\left|A: \operatorname{core}_{G}(T)\right|<\infty$ and since $M$ is faithful $A$ is finite, a contradiction.

Proof of Theorem A. Since $G$ is finitely generated, and the class of polycyclic-byfinite groups is finitely presented, we can suppose that $G$ is not polycyclic-by-finite but that every proper homomorphic image of $G$ is polycyclic-by-finite by [12, Lemma 6.17]. Let $A$ be a non-trivial abelian normal subgroup of $G$. Then $G / A$ is polycyclic-by-finite and we may treat $A$ as a $\mathbb{Z}(G / A)$-module.

Case 1. A has infinite rank. We can suppose that $A=\langle a\rangle^{G}$, where $a \in A, a \neq 1$. Hence, 
$A$ is a cyclic $\mathbb{Z} G$-module. Suppose that $A$ is $\mathbb{Z}$-torsion-free. By [11, Theorem 12.2.7] there is a free abelian subgroup $A_{0}$ of $A$ such that $A / A_{0}$ is a $\pi$-torsion group where $\pi$ is a finite set of primes. If $p \notin \pi$, then $A^{p} \cap A_{0}=A_{0}^{p}$ and so $A_{0} / A_{0}^{p}=A_{0} /\left(A^{p} \cap A_{0}\right) \cong\left(A_{0} A^{p}\right) / A^{p} \subseteq$ $A / A^{p}$. Now as $A$ is $\mathbb{Z}$-torsion free and has infinite rank, $A_{0}$ also has infinite rank. Therefore, $A_{0} / A_{0}^{p}$ is an infinite elementary abelian $p$-group and hence so is $A / A^{p}$. Therefore, $G / A^{p}$ is not polycyclic-by-finite. Our assumption on homomorphic images gives $A^{p}=1$, a contradiction since $A$ is $\mathbb{Z}$-torsion free.

Hence, for some prime $p, A_{p}=\left\{a \in A \mid a^{p}=1\right\} \neq 1$. By assumption, $G / A_{p}$ is polycyclic-by-finite. Hence $A_{p}$ is an infinite elementary abelian $p$-group. We can regard $A_{p}$ as an infinite dimensional $\mathbb{F}_{p} G / A$-module. By Lemma 3.1 there are elements $a \in A_{p}$ and $x \in G$ such that $a \mathbb{F}_{p} X$ is infinite dimensional, where $X=\langle x\rangle$. It follows that the elements $\left\{a x^{n} \mid n \in \mathbb{Z}\right\}$ are linearly independent over $\mathbb{F}_{p}$.

Hence $\langle a\rangle^{X} \cong \bigoplus_{n \in \mathbb{Z}} a^{x^{n}}$, and $H=\langle a, x\rangle \cong C_{p} \sim C_{\infty} \leq G$. Now by [11, Lemma 9.2.8] $k H$ is primitive for any field $k$. Therefore, $k H$ has an infinite dimensional irreducible module and so does $k G$.

Case 2. A has finite rank. Clearly $A$ is not finitely generated. We first reduce to the case where $G=\langle A, x\rangle$ is abelian-by-(infinite cyclic). Let $W$ be an irreducible $\mathbb{Q} G$ submodule of $A \otimes_{\mathbb{Z}} \mathbb{Q}$ and $B=A \cap W$. Then $B$ is finitely generated as a $\mathbb{Z} G$-module but not as an abelian group since $G / B$ is polycyclic-by-finite. We can suppose that $A=B$, so that $A$ is rationally irreducible. By passing to a subgroup of finite index we can suppose $G / C_{G}(A)=\left\langle x_{1}, \ldots, x_{r}\right\rangle$ is abelian. It follows that there is an element $a$ of $A$ and $x=x_{i}$ such that $\langle a\rangle^{\langle x\rangle}$ is not finitely generated as an abelian group. Now the group $\langle a, x\rangle$ is finitely generated and metabelian but not polycyclic-by-finite so we can suppose that $G=\langle a, x\rangle$ and $A=\langle a\rangle^{G}$. As before $A \bigotimes_{\mathbb{Z}} \mathbb{Q}$ is an irreducible $\mathbb{Q}\langle x\rangle$-module. Let $A_{0}$ be a finitely generated subgroup of $A$ of maximum rank such that $A=A_{0}^{\langle x\rangle}$. Let $k$ be a field of characteristic $p \geq 0$. There are now two subcases depending on the structure of $A / A_{0}$.

Suppose first that $\sqrt[p]{A_{0}} / A_{0}$ is infinite. Then by Lemma 2.4 and [8, Theorem 4.2] $k G$ is primitive.

Finally suppose $\sqrt[p]{A_{0}} / A_{0}$ is finite. Since $A / A_{0}$ involves only finitely many primes, there is a maximal ideal $M$ of $k A$ such that $A / M \dagger \cong C_{q^{\infty}}$ where $q \neq p$, by Lemma 2.2. Let $V=k A / M$ and $W=V \otimes_{k A} k G$. We claim that $W$ is an irreducible $k G$-module. Let $C=M \dagger$ and using Lemma 2.3 choose an element $a \in C$. whose conjugates $\left\{a^{x^{r}} \mid r \in \mathbb{Z}\right\}$ lie in infinitely many distinct cosets of $C$ in $A$. Then for all $\left.r \geq 1, C^{\left\langle x^{r}\right.}\right\rangle / C$ is infinite and so $C^{\left\langle x^{\prime}\right\rangle}=A$. Suppose that $W^{\prime}$ is a non-zero submodule of $W$ and that $w=$ $v_{0}+v_{1} x+\ldots+v_{r} x^{r} \in W^{\prime}$ where $v_{i} \in V$, with $v_{0} \neq 0$ and $v_{r} \neq 0$. If $r \geq 1$, then as $C^{\left\langle x^{r}\right\rangle}=A$ there is an element $c$ of $C$ such that either $x^{r} c x^{-r} \notin C$ or $x^{-r} c x^{r} \notin C$. In the first case $v_{0} c=v_{0}$ and $v_{r} x^{r} c \neq v_{r} x^{r}$ while in the second $v_{0} x^{-r} c x^{r} \neq v_{0}$ and $v_{r} x^{r}\left(x^{-r} c x^{r}\right)=v_{r} x^{r}$. A standard 'shortest length' argument shows that $W^{\prime} \cap V \neq 0$. Hence, $V \subseteq W^{\prime}$ since $V$ is an irreducible $k A$-module. However, $W$ is generated by the conjugates of $V$ under $x$ so $W^{\prime}=W$. This completes the proof of Theorem $A$.

Remarks. (1) Part of the motivation for Theorem A lies in obtaining a converse to the result of Jategaonkar [9] that a finitely generated abelian-by-polycyclic group is 
residually finite. For suppose that the group $G$ satisfies the hypothesis of Theorem A, then there is an irreducible $\mathbb{F}_{p} G$-module $A$ such that the split extension $A \sqsupset G$ is finitely generated but not residually finite.

(2) We might ask, as in [3, Questions 2 and 9] for general conditions under which all irreducible $k G$-modules are finite dimensional over $k$. At present only special cases are known. For countable locally finite groups with no elements of order $p$ see [5], [7] and for soluble groups over $\mathbb{C}$, see $[\mathbf{1 5}]$. If $G$ is a finitely generated linear group, then by [16, Theorem 10.16], $G$ is either soluble-by-finite or contains a non-cyclic free subgroup. Since the group algebra of a free group is primitive, it follows from Theorem $A$ that if all the irreducible $k G$-modules are finite dimensional then $G$ is polycyclic-by-finite.

Finally, we remark that if $G$ is a finitely generated infinite $p$-group, and $k$ a field of characteristic $p$, it is not known whether $J(k G)=\omega k G$, see discussion on page 415 of [11]. If this is the case then the only irreducible $k G$-module is the trivial one.

4. Primitive Group Algebras. Throughout this section $A$ will denote a torsion-free abelian group of finite rank and $I$ an ideal of $k A, I \neq k A$. We first outline how the results of Bergman [1] may be extended to this situation. With minor changes we can follow the proof given in [11, Chapter 9] using log subgroups. A log subgroup for $I$ is a subgroup $W$ of $A$ such that for all $\alpha \in I, \alpha \neq 0$ there exist $x, y \in \operatorname{Supp} \alpha$ with $x y^{-1} \in W$ and $x y^{-1} \neq 1$. Whenever these subgroups appear as kernels of valuations as in [11, Lemma 9.3.5] they are necessarily isolated in $A$ since the value group is torsion-free. Thus we can work with isolated $\log$ subgroups.

LEMMA 4.1. Let $A$ be torsion-free abelian of finite rank and $\mathscr{F}=\left\{F_{\alpha} \mid \alpha \in I\right\}$ a collection of finite non-empty subsets of $A$. Let $W=W(\mathscr{F})$ be the set of all isolated subgroups $W$ of $A$ such that $W \cap F_{\alpha} \neq \varnothing$ for all $\alpha$. Then every member of $W$ contains a minimal member, and $W$ has only finitely many minimal members.

Proof. Note that if $\bar{A}$ is any proper torsion-free image of $A$, then $\bar{A}$ has smaller rank than $A$. Hence we can use induction on $\operatorname{rank}(A)$. If $\operatorname{rank}(A)=0$ then $A=\langle 1\rangle$ while if $\operatorname{rank}(A)=1$ the only isolated subgroups of $A$ are $\langle 1\rangle$ and $A$ and the result is trivial in these cases. If $1 \in F_{\alpha}$ for all $\alpha$, then $\langle 1\rangle$ is the unique minimal member of $W$. Suppose that for some $\beta, 1 \notin F_{\beta}=\left\{a_{1}, a_{2}, \ldots, a_{n}\right\}$. Let $W_{i}=\left\{W \in W \mid a_{i} \in W\right\}$. Then we have $W=$ $\mathcal{W}_{1} \cup \mathcal{W}_{2} \cup \ldots \cup \mathcal{W}_{n}$ and it suffices to show that each $W_{i}$ has only finitely many minimal members and that any member of $W_{i}$ contains a minimal member.

Consider $W_{1}$. Let $A_{1}=\sqrt{\left\langle a_{1}\right\rangle}$ the isolator of $\left\langle a_{1}\right\rangle$ in $A$, and let - denote the natural homomorphism $A \rightarrow A / A_{1}=\bar{A}$. If $W \in W_{1}$ then $W \cap F_{\alpha} \neq \varnothing$ for all $\alpha$ and so $\bar{W} \cap \bar{F}_{\alpha} \neq \varnothing$. Moreover, if $W_{1}, W_{2} \in W_{1}$ with $\bar{W}_{1}=\bar{W}_{2}$, then since $a_{1} \in W_{1}, W_{2}$ and the $W_{i}$ are isolated, $A_{1} \subseteq W_{1} \cap W_{2}$ and hence $W_{1}=W_{2}$.

Conversely, if $\bar{W} \in \mathcal{W}(\overline{\mathcal{F}})$ and $W$ denotes the full inverse image of $\bar{W}$ under - then $W$ is isolated in $A$, since $\bar{W}$ is isolated in $\bar{A}$. Hence - gives a one-one correspondence between members of $W_{1}$ and $W(\bar{F})$ and the result follows by induction.

Let $I$ be an ideal of $k A$. We define the rank of $I$ by

$$
\operatorname{rank}(I)=\operatorname{rank}(A)-\max \{\operatorname{rank}(B) \mid I \cap k B=0\} \text {. }
$$


The proof of the next theorem and the corollary may now be adapted from [11, Chapter 9].

THEOREM 4.2. Let $A$ be a torsion-free abelian group of finite rank, and $I \neq k A$ an ideal of $k A$. Then any isolated log subgroup for I contains a minimal isolated log subgroup, and I has only finitely many minimal isolated log subgroups $W_{1}, W_{2}, \ldots, W_{m}$. Furthermore, $\operatorname{rank}(I)=\min \left\{\operatorname{rank}\left(W_{i}\right)\right\}$. Finally if $\alpha$ is any automorphism of $A$, then $W_{1}^{\alpha}, W_{2}^{\alpha}, \ldots, W_{m}^{\alpha}$ are the minimal isolated log subgroups for $I^{\alpha}$.

COROLlary 4.3. Let $A$ be torsion-free abelian of finite rank, and $\Gamma$ a group of automorphisms of $A$. Suppose that $A$ is a $\Gamma$-plinth. If I is a $\Gamma$-invariant ideal of $k A$, then either $I=0$ or $k A / I$ is algebraic over $k$, that is given $a \in A$, there is a polynomial $f(t) \in k[t]$ such that $f(a) \in I$.

We remark that in the situation of the corollary, any prime ideal of $k A$ containing $I$ must be a maximal ideal.

In proving Theorem $\mathrm{B}$, we may assume that $A=A_{0}^{\langle x\rangle}$ where $A_{0}$ is finitely generated.

Lemma 4.4. Let $A_{0}$ be a finitely generated subgroup of $A$ of maximum rank. Let $x$ be an automorphism of $A$ such that no power of $x$ centralises $A$ and $A_{1}=A_{0}^{\langle x\rangle}, G=A J\langle x\rangle$ and $G_{1}=A_{1} J\langle x\rangle$.

(i) Suppose there is a maximal ideal $M$ of $k A_{1}$ such that $\bigcap_{n \in \mathbb{Z}} M^{x^{n}}=0$, then there is a maximal ideal $N$ of $k A$ such that $\bigcap_{n \in \mathbb{Z}} N^{x^{n}}=0$.

(ii) If $k G_{1}$ is primitive then $k G$ is primitive.

Proof. (i) Choose a maximal ideal $N$ of $k A$ containing $M$, by Zorn's Lemma. Then $M=N \cap k A_{1}$. If $I=\bigcap_{n \in \mathbb{Z}} N^{x^{n}} \neq 0$, then $I \cap k A_{1} \neq 0$, since $k A$ is a domain which is integral over $k A_{1}$. However, $I \cap k A_{1}=\bigcap_{n \in Z} M^{x^{n}}=0$. Hence $I=0$.

(ii) This follows by a similar argument using the fact that any non-zero ideal of $k G$ has non-zero intersection with $k A,[11$, Lemma 7.4.9].

As in [4] the primitivity problem for torsion free soluble groups of finite rank may often be reduced to the abelian-by-(infinite cyclic) case.

In the next result and the corollary, $A$ will denote the Zalesskii subgroup of the group $G$ (see [11, section 9.1]). If $I$ is a non-zero ideal of $k G$ then $I \cap k A \neq 0$.

LEMMA 4.5. If $G$ is a torsion-free soluble group of finite rank such that $\Delta(G)=1$, then $\Delta(\langle A, x\rangle)=1$ for some $x \in G$.

Proof. This is essentially the same as the proof of [4, Lemma 4.5]. We merely indicate the modifications that must be made. First we note that for $G$ torsion-free soluble of finite rank, $G$ is nilpotent-by-finite if and only if $G$ is f.c. hypercentral. The crucial point here is that if $B=\Delta(G)$ and $B_{0}$ is a finitely generated subgroup of $G$ such that $B / B_{0}$ is torsion, then $G_{0}=C_{G}\left(B_{0}\right)$ has finite index in $G$ and $G_{0}$ centralizes $B$ since extraction of roots in $B$ is unique. It now follows as in Lemma 4.2 of [4] that $H=G / C_{G}(A)$ is abelianby-finite and we may suppose this group is actually abelian. The action of $G$ on $A$ by conjugation induces a representation of $H$ as matrices with rational entries. If the result is 
false, then by Lemmas 3.2 and 4.4 of [4] each matrix in $H$ has a complex eigenvalue which is a root of unity.

let $V_{0}=A \otimes_{\mathbb{Z}} \mathbb{Q}, V=A \otimes_{\mathbb{Z}} \mathbb{C}$. By Lemma 4.3 of [4] there is a finitely generated subgroup $H_{0}$ of $H$ with $H / H_{0}$ torsion such that $H_{0}$ acts trivially on a non-zero subspace $W$ of $V$. We can assume that

$$
W=V^{H_{0}}=\left\{v \in V \mid v h=v \text { for all } h \in H_{0}\right\} .
$$

Then $W$ is a submodule of $V$. Also by choosing a basis of $\mathbb{C}$ over $\mathbb{Q}$ we see that $W=W_{0} \otimes_{Q} \mathbb{C}$ where $W_{0}=V_{0}^{H_{0}}$. If $H_{1}=C_{H}\left(W_{0}\right)$ then $H / H_{1}$ is isomorphic to a periodic group of matrices over the rational numbers. Hence $H / H_{1}$ is finite, see [12, Part I, p. 85], so there is an integer $k>0$ such that $G^{k} \subseteq C_{G}\left(W_{0}\right)$. The proof may now be completed as in [4, Lemma 4.5].

COROLLARY 4.6. Let $G$ be a torsion-free soluble group of finite rank with $\Delta(G)=1$ and suppose that $G$ is locally polycyclic. If $k$ is a non-absolute field, then $k G$ is primitive.

Proof. By Lemma 4.5 there is an element $x$ in $G$ such that $H=\langle A, x\rangle$ satisfies $\Delta(H)=1$. It suffices to show that $k H$ is primitive. Since $k$ is non-absolute there is an irreducible $k A$-module $V$ which is faithful for $A$. It is easy to see that the induced module $V^{H}=V \otimes_{k A} k H$ is irreducible since $A$ is self-centralising in $H$. Let $I=\operatorname{ann}_{k H}\left(V^{H}\right)$. If $I \neq 0$, then $I \cap k A$ is a non-zero $x$-invariant ideal of $k A$. Since $H$ is locally polycyclic there is a finitely generated $x$-invariant subgroup $A_{0}$ of $A$ such that $A / A_{0}$ is torsion. Then by the argument of Lemma $4.4 J=I \cap k A_{0} \neq 0$ and $J$ is a non-zero $x$-prime ideal of $k A_{0}$ with $\boldsymbol{J} \dagger=1$. This contradicts $[\mathbf{1 4}$, Theorem D].

If $A \otimes_{\mathbb{Z}} \mathbb{Q}$ is irreducible as a $\mathbb{Q}\langle x\rangle$-module we can extend Lemma 2.3.

LEMMA 4.7. Let $x$ be an automorphism of $A$ such that $A=A_{0}^{\langle x\rangle}$ for a finitely generated subgroup $A_{0}$. Suppose that $A$ itself is not finitely generated and that $A \otimes_{\mathbf{Z}} \mathbb{Q}$ is an irreducible $\mathbb{Q}\langle x\rangle$-module. If $B$ is a subgroup of $A$ with $A / B$ infinite then there is an element a of $A_{0}$ whose conjugates $\left\{a^{x^{r}} \mid r \in \mathbb{Z}\right\}$ lie in infinitely many distinct cosets of $B$ in $A$.

Proof. By Lemma 2.3, it is enough to show that $A / B$ has an image isomorphic to $C_{p}^{\infty}$ for some prime $p$. If $\sqrt{B} / B$ is infinite, we observe that $\sqrt{B} / B$ can involve only finitely many primes, so this follows from the structure of Černikov groups, [6, Theorem 19.2]. If $\sqrt{B} / B$ is finite we can suppose that $B=\sqrt{B}$. If in addition $A / A_{0} B$ is infinite the result follows again since $A / A_{0}$ is Črnikov.

Suppose that $A / A_{0} B$ is finite. Then $A / B$ is finitely generated and since $B$ is isolated

$$
A=B \times C \text { with } C \cong A / B
$$

by [6, Corollary 25.3]. Since $A / B$ is infinite, $\operatorname{rank}(B)<\operatorname{rank}(A)$, and so $\bigcap_{n \in \mathbf{Z}} B^{x^{n}}=1$. In fact, some finite intersection is trivial. To see this note that each finite intersection $B^{x^{n_{1}}} \cap B^{x^{n_{2}}} \cap \ldots \cap B^{x_{n}}$ is isolated in $A$ and that $A$ has the minimum condition on isolated subgroups. Therefore $B^{x^{n_{1}} \cap} \cap B^{x_{2}} \cap \ldots \cap B^{x^{n_{r}}}=1$ for finitely many conjugates of $B$. Hence $A$ embeds in $A / B^{x^{n_{1}}} \times A / B^{x_{2}} \times \ldots \times A / B^{x^{n_{r}}}$ which is finitely generated. This contradiction shows that $A / A_{0} B$ cannot be finite if $B$ is isolated. 
Proof of Theorem $C$. We have $A=A_{0}^{\langle x\rangle}$ where $A_{0}$ is a finitely generated subgroup of $A$, and $A$ is an $\langle x\rangle$-plinth. Let $M$ be a maximal ideal of $k A$. We must show that $\bigcap_{n \in \mathbf{Z}} M^{x^{n}}=0$ if and only if $A / M \dagger$ is infinite.

If $|A: M \dagger|=n<\infty$, let $B=\left\{a^{n} \mid a \in A\right\}$ then $B \subseteq M_{\dagger}^{\dagger}$, and since $B$ is characteristic $\bigcap_{n \in Z} M^{x^{n}}$ contains $\omega k B$ and so is non-zero. Now suppose that $A / M \dagger$ is infinite and using Lemma 4.7 choose an element $a$ of $A$ whose conjugates $\left\{a^{x^{r}} \mid r \in \mathbb{Z}\right\}$ lie in infinitely many distinct cosets of $M \dagger$ in $A$. If $I=\bigcap_{n \in \mathbb{Z}} M^{x^{n}} \neq 0$, then by Corollary 4.3, there is a non-zero polynomial $f(t) \in k[t]$ such that $f(a) \in I$. Since $I^{x}=I$ we have $f\left(a^{x^{x}}\right) \in I$ for all $r \in \mathbb{Z}$. However, since the elements $\left\{a^{x^{r}} \mid r \in \mathbb{Z}\right\}$ lie in infinitely many distinct cosets of $M^{\dagger}$, they represent infinitely many distinct elements of the field $k A / M$. In other words the non-zero polynomial $f(t)$ has infinitely many distinct roots in some extension field. This contradiction shows that $I=0$.

Proof of Theorem B. We have a group $G$ of the form $G=\langle A, x\rangle$ where $A$ is an $\langle x\rangle$-plinth, and $G$ is not locally polycyclic. We have to show that $k G$ is primitive for all fields $k$.

By Lemma 4.4, we may assume that $A=A_{0}^{\langle x\rangle}$ where $A_{0}$ is a finitely generated subgroup of $A$. Let $k$ be a field of characteristic $p \geq 0$. If $\sqrt[p]{A_{0}} / A_{0}$ is infinite than $k G$ is primitive by Lemma 2.4 and [8, Theorem 4.2]. On the other hand, if $\sqrt[p]{A_{0}} / A_{0}$ is finite, then since $A / A_{0}$ is infinite, it is not a finite extension of a $p$-group. Therefore, by Lemma 2.2 there is a maximal ideal $M$ of $k A$ such that $A / M \dagger$ is infinite. Hence, $\bigcap_{n \in \mathbb{Z}} M^{x^{n}}=0$ by Theorem $\mathrm{C}$ and again $k G$ is primitive.

Proof of Theorem $D$. We have an $\langle x\rangle$-plinth $A$ and a non-zero $x$-invariant semiprime ideal $I$ of $k A$. We have to show that $A / I \dagger$ is finite and that $I$ is a finite intersection of maximal ideals.

Corollary 4.3 shows that $k A / I$ is algebraic so any prime ideal containing $I$ is maximal. Let $a$ be an element of $A$ and $f(t) \in k[t]$ a polynomial such that $f(a) \in I$. For a maximal ideal $M$ containing $I$ we have $|A: M \dagger|<\infty$ by Theorem $C$. Let $n$ be the least integer such that $a^{n}-1 \in M$, then $\Phi_{n}(a) \in M$ where $\Phi_{n}(t)$ is the $n$th cyclotomic polynomial. Note that $n$ is prime to the characteristic of $k$. If $g(t)$ is a polynomial of least degree such that $g(a) \in M$, then $g(t)$ is irreducible, and $g(t)$ divides $\Phi_{n}(t)$ and $f(t)$ since $\Phi_{n}(a)$ and $f(a)$ belong to $I$. Therefore, for each such integer $n, \Phi_{n}(t)$ and $f(t)$ have a common factor. Since the cyclotomic polynomials $\Phi_{n}(t)$ are relatively prime we obtain only finitely many integers $n$ as $M$ ranges over all maximal ideals containing $I$. Hence, $a^{m}-1 \in I$, for some integer $m$ so that $A / I \dagger$ is periodic. If $I_{0}=I \dagger \cap A_{0}$ then $A_{0} / I_{0}$ is finite, and so $A_{0}^{\langle x\rangle} / I_{0}^{\langle x\rangle}$ has finite exponent so is finite. However, $I_{0}^{\langle x\rangle} \subseteq I \dagger$ since the latter is $\langle x\rangle$-invariant and contains $I_{0}$. Therefore, $A / I \dagger$ is finite and $\operatorname{dim}_{k} k A / I$ is finite. Therefore, there are only finitely many maximal ideals containing $I$, and $I$ is their intersection.

\section{REFERENCES}

1. G. M. Bergman, The logarithmic limit set of an algebraic variety, Trans. Amer. Math. Soc. 157 (1971), 459-469. 

$404-413$

2. K. A. Brown, Primitive group rings of soluble groups, Arch. Math. (Basel) 36 (1981),

3. D. R. Farkas, Group rings, an annotated questionaire, Comm. Alg. 8 (1980), 585-602. 301-315.

4. D. R. Farkas and D. S. Passman, Primitive Noetherian group rings, Comm. Alg. 5 (1978),

5. D. R. Farkas and R. L. Snider, Group algebras whose simple modules are injective, Trans. Amer. Math. Soc. 194 (1974), 241-248.

6. L. Fuchs, Abelian groups (Pergamon, 1960).

7. B. Hartley, Injective modules over group rings, Quart. J. Math. 28 (1977), 1-29.

8. R. S. Irving, Some more primitive group rings, Israel J. Math. 37 (1980), 331-350.

9. A. V. Jategaonkar, Integral group rings of polycyclic-by-finite groups, J. Pure and Applied Algebra 4 (1974), 337-343.

10. I. Kaplansky, Commutative rings (University of Chicago Press, 1974).

11. D. S. Passman, The algebraic structure of group rings (Wiley-Interscience, 1977).

12. D. J. S. Robinson, Finiteness conditions and generalized soluble groups, parts 1 and 2 (Springer-Verlag, 1972).

13. J. E. Roseblade, Group rings of polycyclic groups, J. Pure and Applied Algebra 3 (1974), 307-328.

14. J. E. Roseblade, Prime ideals in group rings of polycyclic groups, Proc. London Math. Soc. 36 (1978), 385-447.

15. R. L. Snider, Soluble groups whose representations are finite dimensional, Abstracts Amer. Math. Soc. (1980), Abstract 775-A6, page 250.

16. B. A. F. Wehrfritz, Infinite linear groups (Springer-Verlag, 1973).

17. B. A. F. Wehrfritz, Groups whose irreducible representations have finite degree I, Math. Proc. Cambridge Philos. Soc. 90 (1981), 411-421.

UNIVERSITY OF WISCONSIN-MADISON

MADISON, WI 53706

USA 